\title{
Educação e saúde no contexto escolar: uma experiência de abordagem lúdica com o tema saúde cardiovascular nos anos iniciais
}

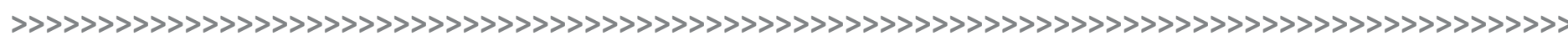

\author{
Simone Lara* \\ Andréia Caroline Fernandes Salgueiro** \\ Jaqueline Copetti*** \\ Karoline Goulart Lanes ${ }^{* * *}$ \\ Robson Luiz Puntel ${ }^{* * * *}$ \\ Vanderlei Folmer*
}

\section{Resumo:}

Devido à relevância da inserção do tema saúde cardiovascular no contexto escolar dos anos iniciais, o presente estudo objetivou apresentar uma proposta de abordagem deste tema através da aplicação de ferramentas lúdicas comalunos dos anos iniciais, por intermédio de estagiários do curso normal.Posteriormente, pode-se verificar a percepção de ambos os grupos sobre a relevância desta proposta. Por fim, um conhecimento mais pertinente sobre o tema foi obtido pelos estagiários do curso normal, bem como conhecer os fatores associados à promoção da saúde do escolar nos anos iniciais, foram algumas das contribuições deste estudo para melhorar o processo de ensino aprendizagem no ensino de ciências e saúde.

\section{Palavras-chave:}

Temas transversais. Saúde. Anos Iniciais. Ferramentas lúdicas. Educação em Ciências.

\section{Abstract:}

Due to the relevance of inserting the topic of cardiovascular health in the elementary school context, this study aimed to present an approach proposal to this theme through the application of ludic tools by trainees in elementary school, and then, to verify the perception of both to the relevance of this proposal. Finally, the most appropriated knowledge about the cardiovascular health theme by the trainees, as well as the factors related to health promotion in elementary school, were some contributions of this study to improve the teaching and learning process in Science and Health Education.

\section{Keywords:}

Cross-cutting themes. Health. Elementary school. Ludic tools. Science education.

\section{Introdução}

Os desafios da educação e da ciência, no que tange ao aprendizado efetivo e significativo de aspectos relacionados à saúde e à qualidade de vida, estão cada vez mais presentes na realidade escolar. Neste contexto, segundo Gil-Perez et al. (2003), as dificuldades encontradas no processo de ensino-aprendizagem estão vinculadas à falta de investimento na educação científica dos professores, que seriam os atores principais a contribuir para pensar e construir um futuro, na direção de uma melhoria da qualidade de vida.
* > Fisioterapeuta, Doutora em Educação e Ciências: Química da Vida e Saúde. Docente na Universidade Federal do Pampa - Campus Uruguaiana. E-mail: simonelara@unipampa.edu.br

** > Fisioterapeuta, Doutoranda em Bioquímica. Universidade Federal do Pampa - Campus Uruguaiana.

E-mail: acfsalgueiro@gmail.com

*** > Professora de Educação Física, Doutora em Educação e Ciências: Química da Vida e Saúde. Docente na Universidade Federal do Pampa.

E-mail: jaquecopetti@yahoo.com.br

**** > Professora de Educação Física, Doutoranda em Educação e Ciências: Química da Vida e Saúde. Universidade Federal de Santa Maria.

E-mail: ktguria@yahoo.com.br

***** > Biólogo, Doutor em Bioquímica Toxicológica. Docente na Universidade Federal do Pampa.

E-mail: robson_puntel@yahoo.com.br

Fisioterapeuta, Doutor em Bioquímica Toxicológica. Docente na Universidade Federal do Pampa.

E-mail: vanderleifolmer@unipampa.edu.br 
De fato, para Fourez (2003), o ensino de ciências deveria ajudar o aluno a compreender o próprio mundo, tendo como principal papel docente a mediação deste processo. No entanto, o ensino de ciências acaba esbarrando em uma realidade descrita por Teixeira (2003) como "rigorosamente marcada pelo conteudismo, excessiva exigência de memorização, descontextualização e ausência de articulação com as demais disciplinas do currículo". Como consequência de todos esses problemas, as aulas são marcadas por informações fragmentadas e sem conexão, impossibilitando a transposição de lacunas que impedem a incorporação do conhecimento de ciências à vida diária (GONZALEZ e PALEARI, 2006).

Outro obstáculo ao ensino de ciências é apontado por Freitas e Reis (2011), quando afirmam que uma das dificuldades encontradas na disseminação dos conhecimentos científicos está no fato de a ciência ser encarada como algo distante da realidade social, ao invés de ser vista como uma produção humana a favor da saúde. Desta forma, cabe aos professores fazerem a intermediação dos processos que visem à socialização da ciência no ambiente escolar, sendo que estes processos devem iniciar-se precocemente, ainda nos anos iniciais, a exemplo do tema saúde, uma vez que este é considerado pelos Parâmetros Curriculares Nacionais (PCN) um tema transversal (BRASIL, 1996), devendo, assim, ser abordado por todos os educadores, dentro das suas especificidades curriculares.

Neste sentido, segundo Machado et al. (2007), a educação em saúde (ES) é uma área do conhecimento que requer uma visão corporificada de distintas ciências, tanto da educação como da saúde. Lopes et al. (2007) complementam que a ES, enquanto processo pedagógico, concebe o homem como sujeito e principal responsável por sua realidade, na qual suas necessidades de saúde são solucionadas a partir de ações conscientes e participativas, organizadas com elementos específicos de seu modo de vida e promovendo mudanças nos atos de saúde e principalmente nas pessoas.

Assim, no presente trabalho, optou-se por trabalhar o tema saúde cardiovascular com professores em formação e, posteriormente, com alunos dos anos iniciais do ensino fundamental, tendo em vista que conhecimentos e comportamentos desenvolvidos por programas de saúde na escola auxiliam as crianças a realizarem escolhas corretas em relação à adoção de hábitos de vida saudáveis (MCGINNIS e DEGRAW, 1991). De fato, as doenças cardiovasculares (DCV) caracterizam-se como doenças crônicas,nas quais o processo de evolução ocorre em grande parte devido a hábitos de vida inadequados. Segundo Nobre et al. (2006) é durante a infância e adolescência que muitos comportamentos de risco e hábitos inadequados são incorporados, entre eles etilismo, tabagismo, sedentarismo e modificações nos padrões alimentares. Este contexto propicia o aparecimento ou o agravo de fatores biológicos de risco para o desenvolvimento destas doenças, sendo de fundamental importância que a educação para a prevenção destes agravos inicie na escola (LANES et al., 2011). Desta forma, Gonzalez e Paleari (2006) afirmam que a educação, na sua dimensão formal do ensino, deve preparar a criança através da aquisição de conhecimentos científicos básicos, para que esta tenha condições de tomar decisões conscientes que impliquem tanto no rompimento de hábitos nocivos, quanto na aquisição de hábitos de vida saudáveis.

Entretanto, para que estes processos de ES aconteçam de forma efetiva no âmbito dos anos iniciais, seus educadores devem estar devidamente preparados para abordar as temáticas de saúde em seus planos de aula. Contudo, Galvão e Praia (2009) destacam que os professores dos anos iniciais não são formados com base numa visão de ciências como a que está sendo, hoje, preconizada e valorizada, o que justifica a realização de projetos visando uma melhor formação e capacitação dos mesmos. Desta forma, optou-se neste estudo por trabalhar com os futuros docentes dos anos iniciais, ou seja, os estudantes do curso normal.

O curso normal geralmente é oferecido nas escolas sob a forma de duas modalidades: médio (com duração de quatro anos para os estudantes com ensino fundamental completo), e pós-médio (com duração de dois anos, para os estudantes que tem formação no ensino médio e desejam ter habilitação também no curso normal), com o objetivo de 
formar educadores para atuar na educação infantil e nos cinco primeiros anos do ensino fundamental. Habitualmente, o contato destes profissionais em formação com as rotinas de sala de aula ocorre ao longo de todo o curso, incluindo atividades de observação e atividades de estágio curricular, o que permite aos estudantes desenvolver discussões sobre a ES no ambiente escolar.

Considerando os alunos dos anos iniciais como público alvo final deste trabalho, fez-se necessário que os temas relacionados à saúde cardiovascular fossem apresentados pelos professores em formação de maneira lúdica, de forma a despertar o interesse, a motivação e o entusiasmo dos alunos, a fim de facilitar a aprendizagem (HOWARD, BELLIN e REES, 2002). Para Soares (2010), a utilização de ferramentas criativas para o ensino de ciências torna-se imperiosa no sentido de buscar uma metodologia capaz de atrair o aluno, de modo que ele compreenda os diversos conhecimentos de maneira lúdica. Conforme apontam Luz e Oliveira (2008), a utilização de ferramentas didáticas alternativas sensibiliza os alunos para o fato de que o conhecimento pode ser gerado ou obtido a partir de diferentes fontes, além de estimular o interesse e a curiosidade dos estudantes pela relação entre conhecimentos derivados da experiência diária, da prática escolar e do conhecimento científico.

De fato, o contexto da ludicidade também proporciona o desenvolvimento da criatividade e das linguagens das crianças (PRANGE e BRAGAGNOLO, 2012), sendo que, para Folmer et al. (2009) a participação do sujeito no processo de construção do conhecimento se faz importante à medida que induz a mudanças de atitude e aumenta a motivação pelo tema em estudo.

Ainda, ponderando-se a ludicidade na abordagem de temas de interesse nos anos iniciais, Muniz (2010) reforça que a utilização de ferramentas lúdicas, a exemplo de jogos e brincadeiras, permite traçar um paralelismo entre o mundo real e o mundo imaginário construído durante e a partir da atividade lúdica, que se traduz em uma representação do mundo sociocultural em que se insere a criança. Cedro e Moura (2004) complementam que a aplicação de atividades lúdicas nas aulas surge como uma opção didático-metodológica que apresenta bons resultados cognitivos, gerando situações problemas desafiadoras para o aluno, e, desta forma, favorecendo o interesse e o aprendizado pelo tema.

Sendo assim e com base no exposto, os objetivos do presente estudo foram:

a. Apresentar uma proposta de abordagem do tema saúde cardiovascular nos anos iniciais, através da aplicação de ferramentas lúdicas no ensino de ciências por estagiários do curso normal;

b. Verificar a percepção dos estudantes dos anos iniciais, bem como a dos estagiários envolvidos no projeto, sobre a aplicação destas temáticas.

\section{Metodologia}

A presente proposta foi aprovada pelo Comitê de Ética da Universidade Federal de Santa Maria (UFSM), e apresentada ao Instituto Estadual de Educação Elisa Ferrari Valls, (Uruguaiana, RS). Os estudantes do curso normal interessados em participar do estudo assinaram um termo de consentimento livre e esclarecido (TCLE) concordando em participar das atividades. Logo, foram incluídas duas turmas de estudantes do curso normal (uma do nível médio e outra do nível pós-médio), totalizando trinta e três estudantes.

Trata-se de uma pesquisa-ação,que visa, além da produção científica, a produção de mudanças na compreensão dos indivíduos envolvidos no processo (O’BRIEN, 1998). As intervenções ocorreram no período compreendido entre março de 2011 e abril de 2012, e as atividades foram desenvolvidas em quatro etapas, sendo: 
a. Inserção do tema saúde cardiovascular - para incluir os temas em saúde cardiovascular no curso normal e capacitar estes estudantes para posterior abordagem aos anos iniciais. Foi entregue aos participantes um cronograma, com tópicos a serem discutidos ao longo dos encontros (tabela 1), para que pudessem pesquisar estes conteúdos previamente. A abordagem desses temas se deu através de rodas de discussão em grande grupo, a fim de facilitar o diálogo e a discussão entre os estudantes do curso normal e os pesquisadores, evitando assim o caráter de aula tradicional expositiva e proporcionando a construção de conhecimento de forma ativa por parte dos alunos. Cabe ressaltar que os pesquisadores eram formados por profissionais da área da saúde e da educação (Fisioterapeutas, Professores de Educação Física e de Ciências Biológicas), que acompanharam os estudantes do curso normal ao longo de todas as etapas do projeto. Cada um dos quatro temas relacionados na tabela 1 foi discutido ao longo de quatro semanas, mediante dois encontros semanais com duração de uma hora e meia cada, totalizando as 16 semanas de trabalho nessa etapa (correspondendo ao período de março a junho de 2011).

Tabela 1 - Temas abordados em saúde cardiovascular

\begin{tabular}{l|l}
\multicolumn{1}{c|}{ Temas } & \multicolumn{1}{c}{ Conteúdos desenvolvidos } \\
\hline $\begin{array}{l}\text { Anatomia e fisiologia } \\
\text { do sistema circulatório }\end{array}$ & $\begin{array}{l}\text { Principais constituintes do sistema cardiovascular } \\
\text { (coração e vasos sanguíneos) e suas respectivas funções }\end{array}$ \\
\hline $\begin{array}{l}\text { Fatores de risco para } \\
\text { as DCV }\end{array}$ & $\begin{array}{l}\text { Inatividade física, padrões alimentares inadequados, uso de drogas, } \\
\text { como o álcool e fumo, obesidade, obesidade central e dislipidemia }\end{array}$ \\
\hline Principais DCV & $\begin{array}{l}\text { Hipertensão arterial sistêmica, Infarto agudo do miocárdio, } \\
\text { Acidente vascular encefálico e Diabetes mellitus }\end{array}$ \\
\hline Prevenção das DCV & $\begin{array}{l}\text { Adoção de estilos de vida saudáveis, em especial no que remete } \\
\text { a alimentação adequada e a prática de exercício físico regular }\end{array}$ \\
\hline
\end{tabular}

Fonte: os autores.

b. Construção de atividades lúdicas no âmbito interdisciplinar - após a etapa de discussão dos temas, os estudantes foram divididos em pequenos grupos e orientados a confeccionarem atividades lúdicas interdisciplinares, a serem aplicadas aos anos iniciais, utilizando como base os temas previamente discutidos em saúde cardiovascular. Considerando que a atividade interdisciplinar caracteriza-se pela intensa troca de saberes e informações entre indivíduos e pelo grau de integração real entre as disciplinas, no interior de um mesmo projeto de pesquisa (JAPIASSU, 1976), reitera-se que, no presente trabalho, os estudantes do curso normal foram estimulados a criar atividades lúdicas com o tema saúde, no aspecto interdisciplinar; ou seja, não somente construindo atividades em saúde que perpassem apenas uma ou outra disciplina, mas, sim, atividades que promovam a integração dos conhecimentos interdisciplinares. Adicionalmente, a equipe de pesquisadores tinha uma formação distinta (profissionais da saúde e da educação), o que também permitiu aos estudantes do curso normal esta troca de informações entre profissionais com formações em diferentes áreas do conhecimento, tendo como base a temática em saúde para a construção das atividades.

A construção destas atividades partiu de uma apresentação simples dos pesquisadores, a respeito dos diversos modelos lúdicos que poderiam ser criados e aplicados às crianças, a exemplo de: palavras cruzadas, criptograma, jogo dos erros, jogo de labirinto, caça palavras, etc. Além disso, os alunos poderiam pesquisar em sites da internet, jornais e revistas, a fim de buscar auxílio para o processo de construção das atividades - sempre acompanhados pelos pesquisadores. Por fim, foram confeccionadas sequências didáticas, constando de atividades lúdicas sobre Saúde Cardiovascular, para posterior aplicação aos alunos nos anos iniciais. 
A etapa II ocorreu no período compreendido entre os meses de agosto a novembro de 2011, totalizando 16 semanas de duração, com dois encontros semanais e duração de uma hora e meia cada. É importante ressaltar que estas intervenções, relacionadas às etapas I e II, aconteceram no ambiente escolar dos estudantes, através de um processo de revezamento dos professores do curso normal, que cederam seus períodos de aula a fim de que o projeto pudesse ser desenvolvido.

c. Aplicação das sequências didáticas - posteriormente, em março de 2012, os estudantes do curso normal passaram para o seu último ano de curso, e assim, nesse momento, poderiam aplicar as atividades construídas no ano anterior para os seus alunos em anos iniciais. Logo, dois estudantes do curso normal pós-médio, que auxiliaram na construção destas atividades e iniciaram seu estágio curricular, selecionaram quatro atividades de suas sequências, a fim de aplicá-las a uma turma de vinte e um alunos do quarto ano do ensino fundamental. As atividades foram aplicadas ao longo de quatro encontros, sendo um encontro por semana com duração de uma hora e meia cada. Cabe ressaltar que antes da entrega das atividades às crianças, os estagiários realizaram atividades práticas e experimentais e explicações prévias sobre o tema, a fim de proporcionar às crianças espaços de discussões entre colegas, esclarecimento de dúvidas, visando melhorar o processo de ensino aprendizagem sobre estas temáticas. Assim, a fim de introduzir estas temáticas, as atividades realizadas foram:

Atividades práticas experimentais - Sistema cardiovascular - explicação sobre a constituição e a função do sistema cardiovascular (coração e principais vasos sanguíneos), através de duas atividades práticas. A primeira consistiu em estimular as crianças a pular durante um minuto e depois verificar o que acontece com o coração e o aumento da frequência cardíaca, levando as crianças a discutirem o porquê destas situações e reações. Na segunda atividade, as crianças foram orientadas a utilizar um canudo para tomar um suco de uva, simulando o transporte de sangue pelos vasos sanguíneos. Nessa situação, foi discutido, entre os pares, o que ocorre com o fluxo sanguíneo no momento de um processo de obstrução vascular (seja no infarto agudo do miocárdio ou no acidente vascular encefálico), simulando situações práticas e perceptíveis às crianças.

Após a introdução destas temáticas por meio das atividades experimentais, as crianças participaram de quatro atividades, envolvendo temas específicos sobre o sistema cardiovascular, descritas a seguir.

"Atividade 1 - sistema cardiovascular (coração e principais vasos sanguíneos), através de aula expositiva dialogada, e a resolução de palavras cruzadas, nas quais as crianças deveriam incluir as palavras que faltavam no diagrama e depois descrever qual palavra-chave iria se formar;

» Atividade 2 - Diabetes mellitus, nutrição, obesidade, desenvolvida através de aula expositiva dialogada sobre os temas, e a resolução de criptograma, no qual as crianças foram orientadas a substituir números por letras, encontrando criptografados alguns dos sintomas desta disfunção, podendo, portanto, descrevê-los sobre os respectivos desenhos.

» Atividade 3 e 4 - Padrões alimentares saudáveis e não saudáveis - desenvolvida através de aula expositiva dialogada sobre os temas, e a resolução de duas atividades. Uma delas envolveu o jogo dos erros e o desenho, onde os alunos deveriam, inicialmente, encontrar os erros entre uma imagem e outra, e, posteriormente, desenhar uma alimentação mais saudável para o menino evitar o ganho de peso. A outra atividade representou uma história em quadrinhos, constituída de duas linhas: a primeira remete a alimentação mais saudável, através da carne de peixe, e a segunda representa o cachorro-quente como a alimentação mais prejudicial à saúde. Assim, as crianças deveriam responder, inicialmente, quais das duas representam a alimentação que prejudica a saúde e depois explicar o porquê de sua resposta. 
Da mesma maneira das etapas anteriores, os pesquisadores do projeto participaram dessas intervenções, observando os seus andamentos e registrando os principais obstáculos/dificuldades de aprendizagem, os questionamentos das crianças e as considerações importantes ponderadas ao longo das atividades.

d. IV. Percepção dos estudantes dos anos iniciais e dos estagiários sobre o projeto - a fim de verificar a percepção destas crianças sobre o desenvolvimento do projeto, estas foram convidadas, ao final de todas as intervenções, a responder por escrito as seguintes questões abertas: 1) Você achou legal participar das atividades sobre saúde? Por quê? e 2) O que você mais aprendeu sobre estas atividades realizadas?

Da mesma forma, os dois estagiários que aplicaram as atividades também responderam por escrito, ao término do projeto, a questão: Qual a sua opinião sobre a abordagem dos temas em saúde cardiovascular (a exemplo destas atividades realizadas) nos anos iniciais?

Cabe ressaltar que a etapa IV foi realizada ao longo de dois encontros no período de abril de 2012, sendo um encontro destinado às crianças participantes do projeto, e o outro aos dois estagiários do curso normal, envolvidos na execução da proposta.

Para a análise dos dados qualitativos foi realizada a análise temática qualitativa segundo Bardin (2004), a fim de categorizar as respostas de acordo com os seus conteúdos.

\section{Resultados e discussão}

O elo entre o lúdico e o ensino já faz parte da didática na educação infantil e tem sido cada vez mais inserido nos anos iniciais com o objetivo de tornar a aula atraente e os alunos mais motivados a aprender. Neste contexto, o estudo abordou o tema saúde cardiovascular de forma lúdica, a fim de melhorar a formação dos estudantes do curso normal, para que pudessem aplicar essas temáticas nos anos iniciais, enquanto educadores.

Para iniciar as atividades, os estagiários do curso normal explicaram às crianças algumas questões que envolviam a constituição e a função do sistema cardiovascular, utilizando como base duas atividades práticas experimentais, a fim de: tornar o processo de ensino aprendizagem mais significativo e lúdico, e favorecer a motivação e o interesse pelo tema (figura 1). Após essa etapa, os alunos realizaram a primeira atividade lúdica, referente às palavras cruzadas sobre a anatomia e a fisiologia do sistema cardiovascular (figura 2).

Figura 1 - Atividades práticas experimentais
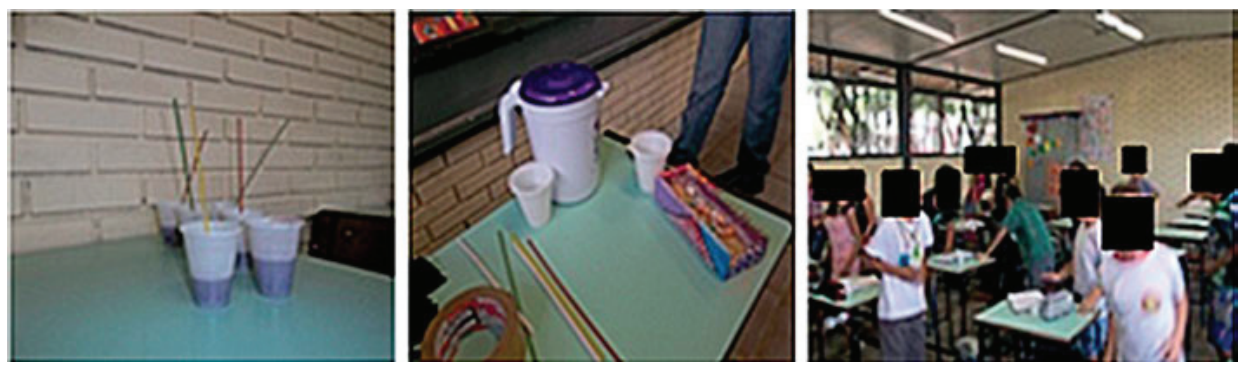

Fonte: os autores, 2012 
Figura 2 - Palavras cruzadas em saúde cardiovascular

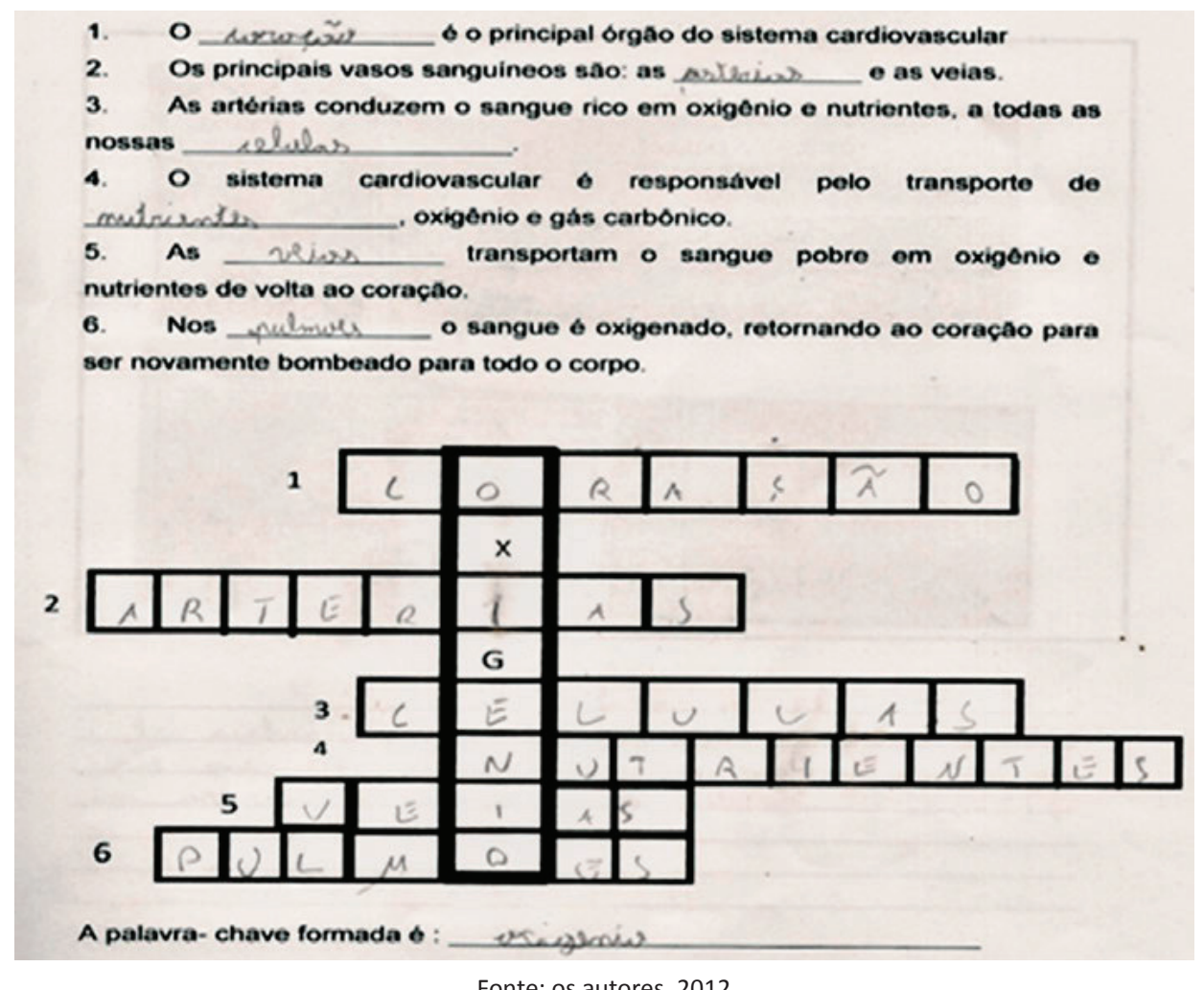

Fonte: os autores, 2012.

Dando continuidade aos temas, os estagiários abordaram a questão do Diabetes mellitus, e após a discussão sobre esta síndrome, os alunos realizaram um criptograma sobre ela (atividade 2, figura 3).

Figura 3 - Criptograma sobre os sintomas do Diabetes mellitus
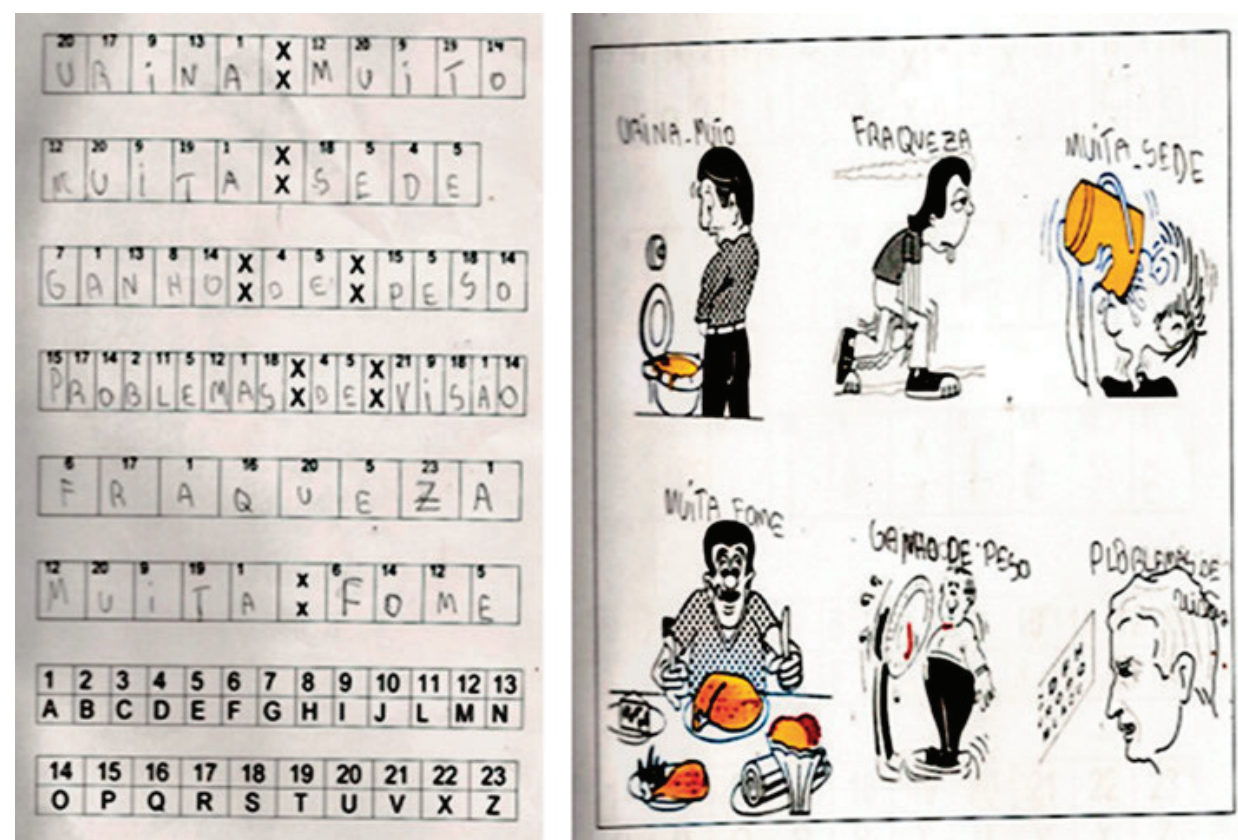

Fonte: os autores, 2012. 
É conhecido que situações lúdicas são essenciais para o estímulo da criatividade, dos relacionamentos sociais e da cognição, podendo facilitar, também, a expressão de emoções, o que é igualmente importante na aprendizagem e na busca de novos conhecimentos. Sendo assim, ao utilizar meios lúdicos, a escola dispõe da possibilidade de instigar o pensamento e enriquecer ambientes com vistas ao desenvolvimento integral da criança (LEITE et al., 2011).

Assim, materiais didáticos como jogos, histórias em quadrinhos, desenhos e caricaturas, são peças-chave nesse contexto, já que podem proporcionar oportunidades impares de investigação e exploração (MOZDENSKI, 2006; CABELLO, ROCQUE e SOUZA, 2010). Nesse sentido, diversos trabalhos reportam uma grande variabilidade de atividades lúdicas para abordar temas relevantes nos anos iniciais, como por exemplo, educação alimentar e nutricional (OLIVEIRA, 2008), educação sexual (VIANNA, 2008), educação no trânsito (DUARTE, et al., 2008), alimentação e obesidade (SOARES, 2010), promoção da saúde bucal (CARCERERI, 2009), benefícios da amamentação (FREIRE et al., 2001), dentre outros. Percebe-se que esses temas, a exemplo do tema saúde cardiovascular retratado neste estudo, são de suma importância para o desenvolvimento integral da criança, uma vez que estão relacionados com a melhoria de suas qualidades de vida. Ainda, através da aplicação de atividades lúdicas, temas de difícil compreensão, a exemplo da fisiologia do sistema cardiovascular (figura 2), bem como os fatores relacionados com o Diabetes mellitus (figura 3), tornam-se mais claros e de melhor entendimento, uma vez que o aspecto lúdico motiva e atrai as crianças, facilitando o processo de ensino aprendizagem no ensino de ciências. Nesse contexto, a participação ativa dos alunos no processo de construção do conhecimento científico, favorece a compreensão do papel da ciência e da tecnologia na sociedade e do desenvolvimento cognitivo, social, político, moral e ético dos estudantes (LEVINSON, 2006; REIS, 2008; SADLER, 2004).

Sabe-se que a obesidade é considerada uma epidemia global e um grave problema de saúde pública não somente no Brasil, mas em vários países, sendo que sua prevalência em crianças e adolescentes vem aumentando nas últimas décadas, acarretando em grandes prejuízos na qualidade de vida destes indivíduos (REILLY et al. 2005).

Desta forma, sabendo das repercussões negativas dos hábitos alimentares inadequados sobre o desenvolvimento da obesidade infantil, o estudo retratou estas questões a fim de criar nas crianças uma conscientização e um maior conhecimento sobre esses temas, nas atividades 3 (jogo dos erros, na figura 4) e 4 (história em quadrinhos, figura 5).

Figura 4 - Jogo dos erros e desenho sobre alimentação.

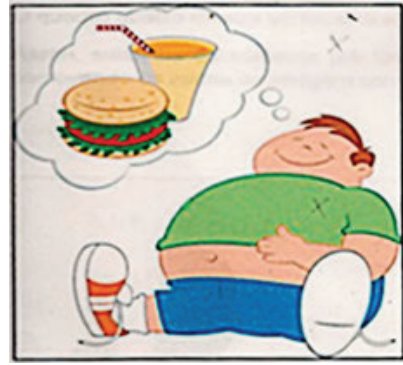

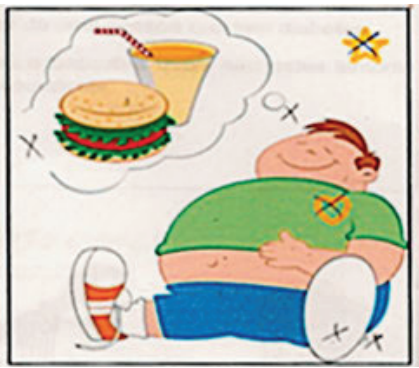

Fonte: os autores, 2012

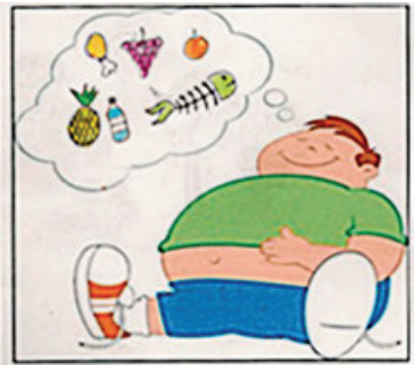

U. 
Figura 5 - Histórias em quadrinhos sobre hábitos alimentares.

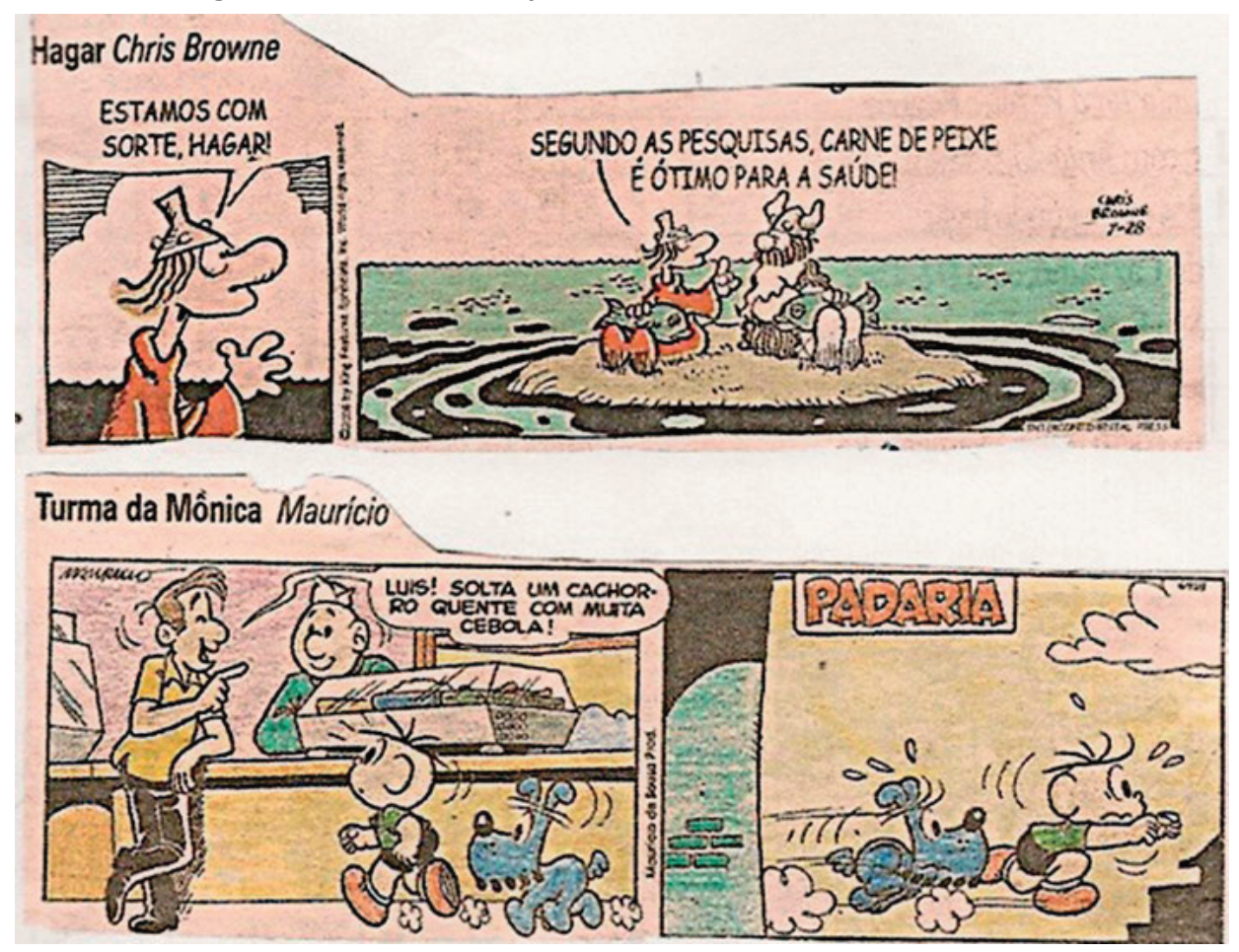

Fonte: Chris Browne e Maurício de Souza, 2012

$\mathrm{Na}$ atividade com as histórias em quadrinhos, reproduzidas acima, as crianças deveriam responder, inicialmente, qual das duas representa a alimentação que prejudica a saúde e depois explicar o porquê de sua resposta. Logo, todos os vinte e um estudantes responderam que o cachorro-quente era o alimento prejudicial à saúde, e os relatos foram estratificados de acordo com as seguintes categorias (BARDIN, 2004):

a. Características do cachorro-quente e os malefícios à saúde - grande parte dos estudantes (dezesseis relatos) respondeu que o cachorro-quente era prejudicial à saúde por conter muita gordura em seus constituintes (maionese, catchup e mostarda) e, assim, esta gordura se acumularia no interior dos vasos sanguíneos, levando ao desenvolvimento de doenças, a exemplo do seguinte relato:

$\mathrm{E}_{8}$ - “O cachorro-quente é uma alimentação inadequada porque contém muita gordura que prejudica a saúde, por exemplo, a maionese que vai entupindo nossas artérias e evita a passagem do sangue, levando a várias doenças".

b. Comparação do cachorro-quente com outros alimentos - três estudantes argumentaram que o cachorro-quente representava a alimentação menos saudável em relação a outros alimentos, a exemplo da carne de peixe e também de frutas, como segue:

$\mathrm{E}_{11}$ - "O cachorro-quente prejudica a saúde e pode causar placas de gorduras nos vasos sanguíneos, prejudicando a saúde, e é menos saudável que as frutas”.

c. Influência da alimentação e do exercício físico para a saúde - dois estudantes atribuíram a importância da aquisição de hábitos de vida saudáveis, como a alimentação adequada e a prática de exercício físico, para promover saúde e prevenir doenças, como observado no relato abaixo:

$\mathrm{E}_{20}$ - "Se não comemos cachorro-quente, que tem gordura, e fazer exercício físico nós não ficamos doentes". 
Considerando o aumento crescente dos índices de escolares obesos, faz-se necessárias intervenções educativas em saúde, abordando os comportamentos nutricionais adequados no contexto escolar (LANES, et al., 2011), visando à prevenção da obesidade nos alunos (GALVÃO e PRAIA, 2009). Contudo, estudos demonstram que as crianças possuem pouco conhecimento sobre aspectos relacionados à nutrição (BIZZO e LEDER, 2005; BRASIL, 2006), o que torna imperativo discutir no ambiente escolar, questões que envolvam os padrões alimentares, a exemplo das figuras 4 e 5 neste estudo. Adicionalmente, cabe salientar que o conhecimento por si só é fundamental para a qualidade de vida das pessoas, mas não suficiente para a modificação de comportamento, no entanto, é fator primordial no auxílio a essa possibilidade de modificação (MALCON et al., 2011).

Por meio da metodologia lúdica utilizada neste trabalho, acredita-se que a aprendizagem em saúde se dê de forma significativa, pois através do brincar, a criança movimenta-se em busca de parceria, e na exploração de objetos, comunica-se com seus pares, se expressa pelas múltiplas linguagens, descobre regras e toma decisões (FRANGIOSI e ROSINI, 2011). Além disso, a brincadeira, independente da sua modalidade, constitui-se de uma rica fonte de estímulo para o desenvolvimento infantil, para o surgimento das interações sociais e para a descoberta das habilidades e potencialidades das crianças (CORDAZZO et al. 2010).

Dessa forma, tal qual apresentada neste estudo, à medida que se desenvolve essas potencialidades, a criança, ao realizar tais atividades lúdicas, reflete sobre questões acerca de sua própria saúde, fato este, segundo Miller (1983: p. 29), relacionado à "alfabetização científica”. Este autor explica que o indivíduo alfabetizado não é aquele que apresenta somente a capacidade de ler e escrever, e sim é culto, erudito, ilustrado. De fato, segundo Batista e Araman (2009), existe a necessidade de implementar novas metodologias que introduzam a criança à alfabetização científica nesse nível de ensino, para que, de acordo com Lorenzetti e Delizoicov (2001), seja capaz de tomar suas decisões de forma consciente, mudando seus hábitos, preservando a sua saúde e exigindo condições dignas para a sua vida e a dos demais seres humanos. Corroborando esse fato, pode-se inferir que o estímulo à tomada de decisão é evidenciado na atividade da figura 5 do presente estudo, em que a criança escolheu qual das duas refeições representa a alimentação não saudável, e, a partir daí, refletiu o porquê de sua escolha. Acredita-se que o estímulo à reflexão, na execução dessa atividade, possa auxiliar a tornar o aluno crítico, e, de acordo com Hurd (1998), capaz de resolver os problemas cotidianos, argumentar, discutir, dialogar, ou seja, prepara-o para tornar-se um cidadão consciente de seus atos e capaz de exercer a cidadania.

No decorrer das atividades, os alunos elencaram vários questionamentos e curiosidades sobre as atividades propostas e as situações de seu cotidiano relacionadas aos temas estudados. Assim, descrevemos algumas destas dúvidas, visualizadas na tabela a seguir.

Tabela 2 - Questionamentos e curiosidades dos estudantes dos anos iniciais ao longo das intervenções realizadas

\begin{tabular}{l|l}
\multicolumn{1}{c|}{ Conteúdos } & \multicolumn{1}{c}{ Questionamentos e dúvidas } \\
$\begin{array}{l}\text { Anatomia do sistema } \\
\text { cardiovascular }\end{array}$ & É veia ou artéria que podemos ver no punho meio azulada? \\
\hline $\begin{array}{l}\text { Fisiologia do sistema } \\
\text { cardiovascular }\end{array}$ & Por que o coração bate rápido depois do exercício? \\
\hline $\begin{array}{l}\text { Diabetes mellitus } \\
\text { Diabetes mellitus }\end{array}$ & O que é insulina? \\
\hline Alimentação e saúde & Onde o pâncreas fica no corpo? \\
\hline
\end{tabular}

Fonte: os autores 
Percebe-se, através dos questionamentos feitos pelas crianças, um envolvimento importante por parte delas ao longo do desenvolvimento do projeto. Verificou-se que os alunos foram capazes de associar o conteúdo dado com situações cotidianas, a exemplo do aumento da frequência cardíaca após um exercício físico, tendo em vista que realizaram tal atividade prática que colocou em evidência esta dúvida. Salientam, Rosa, Rosa e Pecatti (2007) que as atividades experimentais representam uma alternativa metodológica na busca por tornar a aprendizagem em ciências mais significativa para os estudantes nos anos iniciais, além de propiciar o desenvolvimento do pensamento crítico e da imaginação (COSTA, 2011). Ainda, Lima e Garcia (2011) retratam que as aulas práticas diferentes, que motivam os alunos a pensar e construir seus conhecimentos, devem incluir reflexões sobre o funcionamento do corpo humano em situações cotidianas.

Sob esse aspecto, alguns autores colocam que os alunos estarão mais interessados nas temáticas dadas em sala de aula se conseguirem visualizar sua aplicação na vida diária (BURAK e KLÜBER, 2007; CAMARGO, 2007), em especial,os alunos dos anos iniciais, pois as crianças tendem a considerar situações do cotidiano para explicar questionamentos específicos sobre conceitos científicos (DEMCZUK, SEPEL e LORETO, 2007). Freire (1987) complementa que os professores devem criar experiências educativas que considerem a realidade dos alunos e seus saberes construídos cotidianamente, tendo em vista uma formação capaz de transformar a sua realidade. De fato, abordar em sala de aula temas distantes da realidade da criança, os quais ela não consegue fazer a conexão entre os conceitos que vivencia em seu dia-a-dia e aqueles dados na escola, compromete de forma significativa seu aprendizado (ARAÚJO, 2010).

Após a finalização das atividades lúdicas propostas, as crianças foram questionadas a responder as seguintes questões abertas: 1) Você achou legal participar das atividades sobre saúde? Por quê? e 2) O que você mais aprendeu sobre estas atividades realizadas?

Nesta etapa, apenas dezoito estudantes responderam os questionamentos.

Com relação à primeira questão, todos os alunos gostaram de participar do projeto, relatando que as atividades realizadas foram legais. Os relatos foram, novamente, estratificados em três categorias (BARDIN, 2004):

a. Importância destas atividades no cuidado à saúde - onze crianças atribuíram a importância da aplicação destas atividades à aquisição de conhecimentos sobre sua própria saúde, a exemplo do relato:

$\mathrm{E}_{10}$ - "Eu achei legal porque ensina a cuidar da nossa saúde, se alimentar bem e fazer exercício para ficar bem saudável”.

b. Maior conhecimento sobre as temáticas - quatro estudantes responderam que as atividades proporcionaram um maior conhecimento sobre os temas em saúde e $\mathrm{DCV}$, como o funcionamento do coração, os vasos sanguíneos, através do relato:

$\mathrm{E}_{16}$-“Sim porque agente aprendeu muitas coisas sobre o nosso corpo e ficamos sabendo como ele funciona, pois o coração bombeia o sangue para nossas células, para ter energia para nós brincar e se divertir com nossos amigos e nossa família também”.

c. Aprendizado através da ludicidade - três crianças atribuíram a importância destas atividades pela sua característica lúdica, atrativa e interessante, como segue:

$\mathrm{E}_{11}$ - "É divertido porque agente aprende brincando, é interessante e assim aprendemos coisas sobre alimentos saudáveis".

Igualmente, os relatos sobre o que os estudantes mais aprenderam nestas atividades (segunda questão) foram estratificados em três categorias: 
a. Aprendizagem sobre o funcionamento do sistema cardiovascular - sete crianças relataram que os seus maiores aprendizados incluíram os temas envolvendo o funcionamento do coração, o trajeto do sangue através dos vasos sanguíneos, e o transporte de nutrientes até as células; demonstrado no seguinte relato:

$\mathrm{E}_{5}$ - "Eu aprendi que o coração bombeia o sangue para os vasos sanguíneos, e eles transportam os nutrientes até eles entrarem nas células e assim agente consegue energia para pular, brincar e fazer qualquer coisa”.

b. Aprendizagem sobre hábitos de vida (alimentação adequada e exercício físico) - da mesma forma, sete estudantes destacaram que sua maior aprendizagem versou sobre os temas envolvendo a alimentação saudável e a prática de exercício físico para promover saúde e evitar as DCV, como segue:

$\mathrm{E}_{9}$ - "O que eu mais aprendi foi que devemos fazer atividade física e comer coisas saudáveis, e evitar os alimentos com muita gordura que prejudica nossos vasos sanguíneos e nos leva as doenças".

c. Aprendizagem sobre doenças como Diabetes mellitus - quatro relatos retrataram o Diabetes mellitus como o tema que mais aprenderam ao longo das atividades realizadas, demonstrado a seguir:

$\mathrm{E}_{4}$ - "Eu aprendi sobre o Diabetes, que quando nós comemos, tem muita glicose no sangue, e aí vem a insulina que são carrinhos que levam esta glicose para nossas células para ter energia para nós brincar. Só que quando comemos muito doce, fica muita glicose no sangue e aí não tem carrinho suficiente para tirar essa glicose e levar para nossas células, e assim nossas células não vão ter mais energia e vamos ter o Diabetes."

Percebe-se,pelos relatos, que grande parte das crianças atribuiu a importância deste estudo à aquisição de conhecimento sobre sua própria saúde e o autocuidado, em especial, o que remete a uma alimentação adequada e à prática de exercício físico. É interessante verificar que tais percepções sobre vida saudável, especificamente as relacionadas à alimentação e ao exercício físico, são frequentemente relatadas pelas crianças em anos iniciais, a exemplo dos estudos de Moreira e Dupas (2003) e Bottan, Campos e Verwiebe (2008). Além disso, percebe-se nestes trabalhos, que as crianças ainda detêm uma visão muito fragmentada e restrita sobre os fatores que envolvem a saúde, o que torna essencial a abordagem desses temas no ambiente escolar dos anos iniciais, a fim de proporcionar às crianças uma concepção mais ampla sobre os fatores relacionados à saúde. Esse contexto foi percebido através de vários relatos no presente estudo, em que as crianças puderam compreender de forma mais ampla, a origem da energia necessária para a realização de suas atividades de lazer, por meio do funcionamento adequado do sistema cardiovascular.

Apesar de temáticas de difícil compreensão no contexto escolar dos anos iniciais, como as DCV, verificou-se que é possível o entendimento de crianças sobre aspectos que envolvem o Diabetes mellitus, sob uma perspectiva lúdica e uma linguagem apropriada para a faixa-etária, a exemplo do relato que inclui a insulina como uma espécie de "carrinho" que transporta a glicose para o interior das células. Entretanto, apesar da importância desse tipo de intervenção, vários estudos que desenvolvem programas visando à educação sobre o Diabetes mellitus, comumente são direcionados aos próprios portadores da doença (TORRES et al. 2009), ou aos profissionais da saúde (DURAN e COCCO, 2003), no sentido de capacitá-los a lidar com a saúde dos diabéticos, e não sob o aspecto preventivo e educativo.

Após a percepção dos estudantes, os estagiários do curso normal responderam sobre a suas opiniões acerca da aplicação dessas temáticas nos anos iniciais;reproduzidas a seguir: Estagiário ${ }_{1}^{-}$"Refletir sobre a saúde cardiovascular, tornou-se interessante, pois 
as atividades criadas supriram as necessidades dos alunos, bem como a possibilidade de construir novos conceitos sobre o sistema cardiovascular".

Estagiário $_{2}$ - "O tema é uma boa opção para ajudar as crianças a conhecer e a prevenir as doenças cardiovasculares e seus malefícios à saúde. As atividades foram elaboradas para ajudar os alunos a aprender de uma forma mais crítica e criativa, ampliando o conhecimento mais teórico e lúdico sobre as doenças cardiovasculares”.

Assim, percebe-se que os estagiários destacaram a importância da abordagem das temáticas em saúde nos anos iniciais, uma vez que as crianças constroem novos conceitos, percepções e aprendem a cuidar de suas próprias saúdes.

Nessa linha de pensamento, educadores vêm ao longo de anos concordando com a necessidade de a escola assumir um papel de protagonismo com relação ao trabalho de educação nutricional nos anos iniciais, a fim de reduzir os índices de obesidade infantil e prevenir o desenvolvimento das DCV como o Diabetes mellitus (GONÇALVES et al. 2008).

Chia (2010) ressalta que a prevenção primária dessas doenças deve iniciar na infância através do processo de educação para a promoção da saúde cardiovascular, com ênfase na importância da dieta e na manutenção de uma prática regular de atividade física. Da mesma forma, Lanes et al. (2011) salienta a necessidade de detectar os possíveis problemas de saúde mais precocemente e sugerir ações que possam auxiliar na promoção do bem-estar da criança, uma vez que, na medida em que a idade aumenta, os índices são mais preocupantes. Sob este aspecto, Rodrigues, Kolling e Mesquita (2007) retratam que as escolas precisam incorporar de forma precoce temas como saúde (alimentação e exercício físico) para que os indivíduos tenham instrução necessária a fim de prevenir doenças e de promover a saúde, através da adoção de hábitos de vida saudáveis, frutos de uma educação para a saúde do escolar.

Por outro lado, estudos reportam várias dificuldades apontadas pelos educadores, com relação à aplicação e ao desenvolvimento de temas de saúde nas escolas (SILVA, TELAROLLI JÚNIOR e MONTEIRO, 2010; FERNANDES, ROCHA e SOUZA, 2005; SANTOS e BÓGUS, 2007). Neste viés, tais autores colocam que os professores dos anos iniciais carecem de conhecimento teórico e experiência prática, sentem-se inseguros para criar novas atividades em educação em ciências, e recebem pouca orientação e suporte para mudanças (BENCZE e HODSON, 1999; HARLEN e HOLROYD, 1997; GARCÍA BARROS e MARTÍNEZ-LOSADA, 2001).

Desta forma, faz-se necessárias profundas modificações curriculares ao longo da formação dos docentes, seja o licenciando em pedagogia ou o estudante do curso normal, além de oficinas e cursos de formação continuada, a fim de capacitá-los e prepará-los para a abordagem de temas relevantes, a exemplo da saúde, no contexto escolar dos anos iniciais. De fato, de acordo com Franco e Libâneo (2007), a ciência pedagógica deve promover, na formação dos pedagogos, a articulação da teoria educacional com a prática educativa, a fim de reduzir práticas alienantes da escola e da sociedade e, assim, encaminhar a sociedade para processos cada vez mais humanizadores. Adicionalmente, é relevante a colaboração entre profissionais de diversas áreas, evitando assim a disseminação de informações equivocadas e analogias incorretas sobre temáticas específicas. Os autores colocam, também, que melhorando a formação dos pedagogos, possibilita-se a elevação da qualidade cognitiva da aprendizagem dos alunos, com vistas a formar indivíduos mais cultos, bem como cidadãos mais atuantes dos benefícios culturais e tecnológicos promovidos pelo desenvolvimento social e econômico.

Sendo assim, é vital que os futuros educadores dos anos iniciais abordem em suas aulas, por meio de um ensino lúdico e atrativo, questões que levem a criança a refletir e a aprender hábitos de vida saudáveis, a fim de que possam efetivamente melhorar a sua qualidade de vida e a de sua comunidade. Para tal, esses educadores devem ser formados e atualizados para trabalhar os temas de saúde em suas aulas (FERNANDEZ 
e SILVA, 2008), destacando-se, assim, a importância do presente estudo como forma de viabilizar esse aprendizado.

\section{Considerações finais}

Considerando a relevância da inserção do tema saúde cardiovascular no contexto escolar dos anos iniciais, nosso estudo possibilitou, por meio da capacitação de estagiários do curso normal, a aplicação de atividades lúdicas direcionada aos anos iniciais, objetivando promover um maior conhecimento por parte das crianças sobre aspectos específicos envolvendo o tema saúde.

Dessa forma, ficou evidente a participação efetiva das crianças, através de seus questionamentos e curiosidades, de seus envolvimentos nas atividades lúdicas e práticas e em seus relatos. Essa participação e interesse se fazem relevantes, uma vez que as crianças se apresentam mais receptivas a novas aprendizagens, e tendem a incorporar hábitos de vida que perduram ao longo da vida adulta, sendo vital a abordagem de estilos de vida saudáveis de forma precoce no contexto escolar. Nomeadamente, houve um engajamento importante dos estagiários do curso normal que conduziram o trabalho, de forma a manter as crianças motivadas pela aprendizagem em saúde. De fato, os estagiários mostraram-se seguros e motivados para abordar o tema com as crianças, possivelmente pelo fato de que foram os principais agentes envolvidos no processo de aquisição de conhecimento sobre as temáticas, participando efetivamente de todas as etapas do trabalho.

Salientamos, portanto, a importância da inserção de projetos e oficinas de capacitação, além de cursos de formação continuada direcionados aos docentes dos anos iniciais, para que reflitam sobre suas práticas pedagógicas e retratem em suas aulas temas de relevância social, de acordo com os preceitos dos PCN. Acreditamos que, por meio dessas ações, o educador poderá proporcionar um ensino mais contextualizado, lúdico, considerando as situações cotidianas do aluno, tornando a aprendizagem em ciências mais significativa.

Assim, um conhecimento mais pertinente sobre o tema saúde cardiovascular pelos estagiários do curso normal, bem como os fatores associados à promoção da saúde do escolar nos anos iniciais, foram algumas das contribuições do presente estudo para melhorar o processo de ensino aprendizagem no ensino de ciências e saúde.

\section{Agradecimentos}

Agradecemos a CAPES, FAPERGS e CNPq, aos professores: Leandro da Silva Teixeira, Zaira Pereira Schunemann, Suely Zenore Caputo Aymore, Khatia Janette Saldanha da Silva, Cleiva Leite de Moraes, Luciana Genro Bilhalba, Laura Barrenche Lhamby, Elizete Vaz Alberto; e aos diretores: Noeli de Oliveira Carrilho e Maria Cristina Silveira Galvão Gomes, do Instituto Estadual de Educação Elisa Ferrari Valls, pela dedicação e colaboração que apresentaram ao longo do estudo.

\section{Referências}

ARAÚJO, Isabel Cristina de Almeida. Percepções de alunos e professores do $5^{\circ}$ e $6^{\circ}$ anos do ensino fundamental: reflexões das práticas docentes. In: COLÓQUIO INTERNACIONAL EDUCAÇÃO E CONTEMPORANEIDADE, 4., Laranjeiras, Sergipe, 2010. Disponível em: <http://www.educonufs. com.br/ivcoloquio/cdcoloquio/eixo_08/e8-50.pdf> Acesso em: 3 dez. 2010.

BARDIN, Laurence. Análise de conteúdo. Lisboa: Edições, 2004. 
BATISTA, Irinéa de Lourdes; ARAMAN, Eliane Maria de Oliveira. Uma abordagem histórico-pedagógica para o ensino de ciências nas séries iniciais do ensino fundamental. Revista Electrónica de Enseñanza de las Ciencias, v. 8, n. 2, p. 466-489, 2009.

BENCZE, Larry; HODSON, Derek. Changing practice by changing practice: toward a more authentic science and science curriculum development. Journal of Research in Science Teaching, v. 36, n. 5, p. 521-539, 1999.

BIZZO, Maria Letícia Galluzzi; LEDER Lidia. Educação nutricional nos parâmetros curriculares nacionaispara o ensino fundamental. Revista de Nutrição, Campinas, v. 18, n. 5, p. 661-667, 2005.

BOTTAN, Elisabete Rabaldo; CAMPOS, Luciane; VERWIEBE, Ana Paula Schwarz. Significado do conceito de saúde na perspectiva de escolares do ensino fundamental. Revista Brasileira em Promoção da Saúde, Fortaleza,v. 21, n. 4, p. 240-245, 2008.

BRASIL. Ministério da Educação e do Desporto. Secretaria da Educação do Ensino Fundamental, Parâmetros Curriculares Nacionais: ensino fundamental. Brasília: MEC/SEF, 1996.

BRASIL. Resolução FNDE n 32 de 10 de agosto de 2006. Estabelece as normas para execução do Programa Nacional de Alimentação. Brasília: Fundo Nacional de Desenvolvimento da Educação, 2006.

BURAK, Dionísio; KLÜBER, Tiago Emanuel. Modelagem Matemática na Educação Básica: uma trajetória. In: ENEM - ENCONTRO NACIONAL DE EDUCAÇÃO MATEMÁTICA, 9.,2007, Belo Horizonte. Anais... Belo Horizonte: UNI-BH, 2007. Disponível em: <http://www.dionisioburak. com.br/IX\%20ENEM\%20-\%20Dionisio.pdf> Acesso em: 22 dez. 2007.

CABELLO, Karina; ROCQUE, Lucia de La; SOUZA, Isabela Cabral Félix de. Uma história em quadrinhos para o ensino e divulgação da hanseníase. Revista Electrónica de Enseñanza de las Ciencias, v. 9, n. 1, p. 225-241, 2010.

CARCERERI, Daniela Lemos. Promoção de saúde na escola: caderno 1 - Saúde Bucal. Departamento de odontologia, Universidade Federal de Santa Catarina, 2009. Disponível em: <http://ca.ufsc.br/ saude_bucal/cadernol.pdf>. Acesso em: 21 dez. 2009.

CAMARGO, Marco Antonio de. Telecurso 2000: uma análise da articulação da matemática escolar e do cotidiano nas tele-aulas. 2007. 150f. Dissertação (Mestrado em Educação), Programa de pósgraduação em Educação, Universidade São Francisco, Itatiba, 2007. Disponívelem:<http://www.usf. edu.br/itatiba/mestrado/educacao/uploadAddress/Dissertacao_Marco_Antonio_Camargo[1557]. pdf $>$. Acesso em: 25 dez. 2007.

CEDRO, Wellington Lima; MOURA, Manoel Oriosvaldo de. O espaço de aprendizagem e a atividade de ensino: o clube de matemática. In: ENCONTRO NACIONAL DE EDUCAÇÃO MATEMÁTICA,8.,2004, Recife. Anais... Recife, 2004. Disponível em: <http://www.sbem.com.br/files/ viii/pdf/02/CC78728770153.pdf>. Acesso em: 12 dez. 2004.

CHIA, Michael. Pedometer-assessed physical activity of Singaporean youths. Preventive Medicine, v. 50, p. 262-4, 2010.

CORDAZZO, Sheila Tatiana Duarte et al. Brincadeira em escola de ensino fundamental:um estudo observacional. Interação em Psicologia, Curitiba, v. 14, n. 1, p. 43-52, 2010.

COSTA, Gilcilene Dias da. Curricularte: experimentações pós-críticas em educação. Revista Educação e Realidade, Porto Alegre, v. 36, n. 1, p. 279-293, jan./abr., 2011.

DEMCZUK, Oxana Marucya; SEPEL, Lenira Maria Nunes; LORETO, Elgion Lucio Silva. Investigação das concepções espontâneas referentes a ciclo de vida e suas implicações para o ensino nas series iniciais. Revista Electrónica de Enseñanza de las Ciencias, v. 6, n. 1, p. 117-128, 2007.

DURAN, Erika Christiane Marocco; COCCO, Maria Inês Monteiro. Software educativo sobre diabetes mellitus para profissionais de saúde: etapas de elaboração e desenvolvimento. Revista Latino-americana de Enfermagem, Ribeirão Preto, SP, v. 11, n. 1, p. 104-7, 2003.

DUARTE, Regina Maria et al. A criança no trânsito. Secretaria de Justiça e Segurança Pública, Conselho Estadual de Trânsito, Mato Grosso do Sul, 2008. Disponível em: <http://www.cetran. ms.gov.br/controle/ShowFile.php?id=21656>. Acesso em: $5 \mathrm{dez} .2008$.

FERNANDES, Marcos Henrique; ROCHA, Vera Maria; SOUZA, Djanira Brasilino de. A concepção sobre saúde do escolar entre professores do ensino fundamental ( $1^{\mathrm{a}}$ a $4^{\mathrm{a}}$ séries). História, Ciências, Saúde-Manguinhos, v. 12, n. 2, p. 283-91, 2005. 
FERNANDEZ, Patrícia Martins; SILVA, Denise Oliveira e. Os grupos alimentares por professores de $1^{\mathrm{a}}$ a $4^{\mathrm{a}}$ série: a necessidade de atualização dos conceitos. Ciência \& Educação, Bauru, SP, v. 14, n. 3, p. 451-66, 2008.

FOUREZ, Gerard. Crise no Ensino de Ciências? Revista Investigações em Ensino de Ciências, v. 8, n. 2, p. 109-123, 2003.

FOLMER, Vanderlei; BARBOSA, Nilda de Vargas; SOARES, Félix Antunes; ROCHA, João Batista Teixeira da Rocha. Experimental activities based on ill-structured problems improve Brazilian school students' understanding of the nature of scientific knowledge. Revista Electrónica de Enseñanza de las Ciencias, v. 8, n. 1, p. 232-254, 2009.

FRANCO, Maria Amélia Santoro; LIBÂNEO, José Carlos. Elementos para a formulação de diretrizes curriculares para cursos de pedagogia. Cadernos de Pesquisa, v. 37, n. 130, p. 63-97, 2007.

FRANGIOSI, Gabriela; ROSINI, Alessandro. Marco. A importância dos jogos e brincadeiras na prática educativa: séries iniciais. Revista Inovação Tecnológica, v. 1, n. 1, p. 59-64, 2011.

FREIRE, Maria Teresa Marins et al. Visão infantil do aleitamento materno: Projetode Aleitamento Materno. Pontifícia Universidade Católica do Paraná, Pró-Reitoria Comunitária e de Extensão, Programa de Ação Comunitária e Ambiental, Curitiba, 2001. Disponível em: <http://www.pucpr. br/servicos/programas_saude/palma/arquivos/visaoinfantil.pdf $>$. Acesso em: 21 dez. 2001.

FREIRE, Paulo. Pedagogia do oprimido. 17. ed. Rio de Janeiro: Paz e Terra, 1987.

FREITAS, Jairo Dias de; REIS, Silvia Barreiros dos. Ensino de ciências e formação profissional em saúde de nível médio: representações sociais e visões de ciência. Ciência \& Educação, Bauru, SP, v. 17, n. 3, p. 693-704, 2011.

GALVÃO, Viviane Souza; PRAIA, João Feliz. Construir com os professores do $2^{\circ}$ ciclo práticas letivas inovadoras: um projeto de pesquisa sobre o ensino do tema curricular 'alimentação humana'. Ciência \& Educação, Bauru, SP, v. 15, n. 3, p. 631-645, 2009.

GARCÍA BARROS, Susana; MARTÍNEZ-LOSADA, Cristina. Qué actividades y qué procedimientos utiliza y valora el profesorado de educación primaria. Enseñanza de las Ciencias, v. 19, n. 3, p. 433-452, 2001.

GIL-PEREZ, Daniel et al. A educação científica e a situação do mundo: um programa de atividades dirigido a professores. Ciência \& Educação, Bauru, SP, v. 9, n. 1, p. 123-146, 2003.

GONÇALVES, Fernanda Denardin, et al. Health promotion in primary school. Interface - Comunicação, Saúde, Educação, v. 12, n. 24, p. 181-92, 2008.

GONZALEZ, Fabiana Gaspar; PALEARI, Lucia Maria. O ensino da digestão-nutrição na era das refeições rápidas e do culto ao corpo. Ciência \& Educação, Bauru, SP, v. 12, n. 1, p. 13-24, 2006.

HARLEN, Wynne; HOLROYD, Colin. Primary teachers' understanding of concepts of science: impact on confidence and teaching. International Journal of Science Education, v. 19, n. 1, p. 93-105, 1997.

HOWARD, Justine; BELLIN, Wynford; REES, Val. Eliciting Children's Perceptions of Play and Exploiting Playfulness to Maximise Learning in the Early Years Classroom. In: ANNUAL CONFERENCE OF THE BRITISH EDUCATIONAL RESEARCH ASSOCIATION, 2002, England. Disponível em: <http://www.leeds.ac.uk/educol/documents/00002574.htm>. Acesso em: 10 dez. 2002.

HURD, Paul DeHart. Scientific literacy: new mind for a changing world. In: Science \& Education, Stanford, USA, v. 82, n. 3, p. 407-416, 1998.

JAPIASSU, Hilton. Interdisciplinaridade e patologia do saber Rio de Janeiro: Imago, 1976.

LANES, Karoline Goulardt, et al. Sobrepeso e obesidade: implicações e alternativas no contexto escolar. Revista Científica Eletrônica Ciências \& Ideias, v. 3, n. 1, 2011.

LEITE, Maria Letícia Felicori Tonelli e Teixeira. et al. Jogos digitais: uma abordagemeducacional à luzda epistemologia genética. Cadernos do Aplicação, Porto Alegre, v. 24, n. 1, p. 125-56, 2011.

LIMA, Daniela Bonzanini de; GARCIA, Rosane Nunes. Uma investigação sobrea importância das aulas práticasde biologia no ensino médio. Cadernos do Aplicação, Porto Alegre, v. 24, n. 1, p. 201-24, 2011.

LEVINSON, Ralph. The use of narrative in supporting the teaching of socio-scientific issues: a study of teachers' reflections. Interacções, Santarém, Portugal, v. 2, n. 4, p. 24-41, 2006. 
LORENZETTI, Leonir; DELIZOICOV, Demétrio. Alfabetização científica no contexto das séries iniciais. ENSAIO - Pesquisa em Educação em Ciências, Belo Horizonte, v. 3, n. 1, p. 37-50, 2001.

LOPES, Elisabeth de Fátima da Silva. et al. Educação em saúde: um desafio para a transformação da práxis no cuidado em enfermagem. Revista do Hospital das Clínicas de Porto Alegre, Porto Alegre, v. 27, n. 2, p. 25-27, 2007.

LUZ, Maurício; OLIVEIRA, Maria de Fátima Alves de. Identificando os nutrientes energéticos: uma abordagem baseada em ensino investigativo para alunos do Ensino Fundamental. Revista Brasileira de Pesquisa em Educação em Ciências, Belo Horizonte, v. 8, n. 2, 2008.

MACHADO, Maria de Fátima Antero Sousa, et al. Integralidade, formação de saúde, educação em saúde e as propostas do SUS: uma revisão conceitual. Ciência \& Saúde Coletiva, v. 12, n. 2, p. 335-342, 2007.

MALCON, Maura Cavada; MENEZES, Ana Maria Baptista; ASSUNÇÃO, Maria Cecília Formoso; NEUTZLING Marilda Borges; CHALLAL Pedro et al. Efetividade de uma intervenção educacional em tabagismo entre adolescentes escolares. Rev. Bras. Epidemiologia, São Paulo, v. 14, n. 1, p. 63-72, 2011.

MCGINNIS, Michael; DEGRAW, Christopher. Healthy Schools 2000: creating partnerships for the decade. Journal of School Health, v. 61, n. 7, p. 192-7, 1991.

MILLER, JonD. Scientific literacy: a conceptual and empirical review. Daedalus, v. 112, n. 2, p. 29-48, 1983.

MOREIRA, Patrícia Luciana; DUPAS, Giselle. Significado de saúde e de doença na percepção da criança. Revista Latino-americana de Enfermagem, v. 11, n. 6, p. 757-62, 2003.

MOZDZENSKI, Leonardo Pinheiro. A cartilha jurídica: aspectos sócio-históricos, discursivos e multimodais. 2006. Dissertação (Mestrado em Lingüística) - Programa de Pós-Graduação em Letras da Universidade Federal de Pernambuco, Recife, 2006. Disponível em: <http://www.pgletras. com.br/2006/dissertaoes/diss-leonardo-mozdzenski.pdf>. Acesso em: 05 dez. 2006.

MUNIZ, Cristiano Alberto. Brincar e jogar: enlaces teóricos e metodológicos no campo da educação matemática. Belo Horizonte: Autêntica, 2010.

NOBRE, Moacyr Roberto Cuce, et al. Prevalências de sobrepeso, obesidade e hábitos de vida associados ao risco cardiovascular em alunos do ensino fundamental. Revista da Associação Médica Brasileira, São Paulo, v. 52, n. 2, p. 118-24, 2006.

O'BRIEN, Rory. An overview of the methodological approach of action research. 1998. Disponível em: <http://www.web.ca/ robrien/papers/arfinal.html>. Acesso em: 12 out. 1998.

OLIVEIRA, Kathleen Sousa. Evaluation of the 2005 "Criança saudável - educação dez" teaching material. Interface - Comunicação, Saúde, Educação, Botucatu, SP, v. 12, n. 25, p. 401-10, 2008.

PRANGE, Bruna; BRAGAGNOLO, Regina Ingrid. As Singularidades das Crianças Pequenas Expressas nas suas Brincadeiras. Revista Educação e Realidade, Porto Alegre, v. 37, n. 1, p. 251-271, jan./abr. 2012.

REILLY, John; ARMSTRONG, Julie; DOROSTY, Ahmad; EMMET, Pauline; STEER, Colin; SHERRIFF, Andrea. Early life risk factors for obesity in childhood: cohort study. BMJ, Londres, Reino Unido, v. 330, p. 1-7, 2005.

REIS, Pedro. A escola e as controvérsias sociocientíficas: perspectivas de alunos e professores. Lisboa: Escolar Editora, 2008.

RODRÍGUEZ, Carlos Arteaga; KOLLING, Marcelo Garcia; MESQUITA, Peri. Educação e Saúde: um Binômio que Merece Ser Resgatado. Revista Brasileira de Educação Médica, Rio de Janeiro, v. 31, n. 1, p. 60- 66, 2007.

ROSA, Cleci Werner da; ROSA, Álvaro Becker da; PECATTI, Claudete. Atividades experimentais nas séries iniciais: relato de uma investigação. Revista Electrónica de Enseñanza de las Ciencias, v. 6 , n. 2, p. 263-274, 2007.

SADLER, Troy. Informal reasoning regarding socioscientific issues: a critical review of research. Journal of Research in Science Teaching, v. 41, n. 5, p. 513-536, 2004. 
SANTOS, Kátia Ferreira dos; BÓGUS, Cláudia Maria. A percepção de educadores sobre a escola promotora de saúde: um estudo de caso. Revista Brasileira de Crescimento e Desenvolvimento Humano, São Paulo, v. 17, n. 3, p. 123-133, 2007.

SILVA, Andréia Cristina de Almeida; TELAROLLI JÚNIOR, Rodolpho; MONTEIRO, Maria Iolanda. Analisando conhecimentos e práticas de agentes educacionais e professoras relacionados à alimentação infantil. Ciência \& Educação, Bauru, SP, v. 16, n. 1, p. 199-214, 2010.

SOARES, Max Castelhano. Uma proposta de trabalho interdisciplinar empregando os temas geradores alimentação e obesidade. 2010. Dissertação de Mestrado (Mestrado em Educação em ciências) - Programa de pós-graduação em educação em ciências, Universidade Federal de Santa Maria, Santa Maria, 2010. Disponível em: <http://www.pgletras.com.br/2006/dissertaoes/diss-leonardomozdzenski.pdf >. Acesso em: $06 \mathrm{dez} .2010$.

TEIXEIRA, Paulo Marcelo. A educação científica sob a perspectiva da pedagogia histórico-crítica e do movimento C.T.S. no ensino de ciências. Ciência \& Educação, Bauru, SP, v. 9, n. 2, p. 177-190, 2003.

TORRES, Heloisa Carvalho, et al. O processo de elaboração de cartilhas para orientação do auto cuidado no programa educativo em diabetes. Revista Brasileira de Enfermagem, Brasília, v. 62, n. 2, p. 312-6, 2009.

VIANNA, Tiago Fernandes. A sexualidade em cartilhas educativas oficiais: uma análise cultural. 2008. Monografia (Trabalho de Conclusão de curso em Ciências Biológicas) - Faculdade de Ciências Biológicas, Universidade Federal de Santa Catarina, Florianópolis, 2008. Disponível em: <http:// www.cienciasbiologicas.ufsc.br/TCC-BIOLOGIA-UFSC/TCCTiagoFVianaBioUFSC-08-1.pdf>. Acesso em: 21 mar. 2011. 